\title{
XMM Observations of Metal Abundances in Galaxy Clusters
}

\author{
L. Lovisari, S. Schindler, W. Kapferer
}

\begin{abstract}
The hot gas that fills the space between galaxies in clusters is rich in metals. Due to their large potential well, galaxy clusters accumulate metals over the whole history of the cluster, and retain important information on cluster formation and evolution.

We derive detailed metallicity maps for a sample of 5 clusters, observed with XMM-Newton, to study the distribution of metals in the Intra-Cluster Medium (ICM). We show that even in relaxed clusters the distribution of metals shows many inhomogeneities with several maxima separated by low metallicity regions. We also found a deviation from the expected temperature-metallicity relation.
\end{abstract}

Keywords: galaxies, cluster, general - galaxies, abundances - X-ray, galaxies, cluster, intergalactic Medium.

\section{Introduction}

Since the first X-ray observations of the $7 \mathrm{keV}$ iron line feature in the 1970's we have known that the intra-cluster medium (ICM) contains not only primordial elements but also heavy elements. As heavy elements are only produced in stars which reside mainly in galaxies, the enriched material must have been ejected into the ICM by the member galaxies. Due to the large potential wells of galaxy clusters they retain all the enriched material. This makes them excellent laboratories for the study of nucleosynthesis and of the chemical enrichment history of the universe. Because the gas transfer affects the evolution of galaxy and galaxy cluster, it is important to know when and how the enrichment takes place.

The components in a galaxy cluster interact with each other in many different ways. A study of the distribution of the ejected metals can therefore give us important information on the mechanisms that transported the enriched gas into the ICM.

Several processes have been proposed for explaining the observed enrichment in the ICM: rampressure stripping, galactic winds, galaxy-galaxy interactions, AGN outflows, intra-cluster supernovae, and others. Simulations show an inhomogeneous distribution of the metals independent of the enrichment processes [1]. Nevertheless, AGN outflows and galaxy-galaxy interactions can add metals to the ICM [2]. Simulations suggest that the metal enrichment of the ICM is primarily due to galactic winds and ram-pressure stripping. A detailed comparison between the enrichment due to galactic winds and due to ram-pressure stripping revealed that these two processes yield different metal distributions and a different time dependence of the enrichment [3]. In massive clusters, ram-pressure stripping provides a much more centrally concentrated distribution than galactic winds. This is because galactic winds can be suppressed in the cluster center while ram-pressure stripping is most efficient there due to the fact that the ICM density as well as the galaxies velocities are larger in the cluster center [3].

X-ray spectra are the only measure for the metallicity of the ICM. The metallicity is derived mainly by measuring the equivalent width of the iron line once the continuum (almost entirely due to thermal bremsstrahlung) is known. With the first generation of satellites it was just possible to determine the radial metallicity profiles [4]. With deep observations of bright clusters of galaxies by the Chandra and XMM-Newton satellites it is now possible to extract the metallicities in certain regions of a galaxy cluster and to construct X-ray weighted metallicity maps $[5,6,7,8,9,10,11,12,13,14]$.

\section{Metallicity maps}

In order to study the distribution of metals, we prepared adaptively binned abundance maps for a sample of five clusters (Centaurus, A2029, A496, S159-03 and Hydra A). Appropriately cleaned data sets for all three XMM-Newton EPIC cameras, with point sources removed, were used to create the source spectra. A high statistic is required in order to obtain a metallicity measurement with good accuracy. Thus, to ensure an acceptable error also in the outskirts of the clusters we set a minimum required count number ( $\sim 5000$ source counts per region in the $0.3-10 \mathrm{keV}$ band) for proceeding with the spectral fit. In order to model the emission from a single (or multi) temperature plasma we fit the spectra with an APEC (+APEC) model multiplied by the Galactic column density fixed at the Galactic values. The spectral re- 

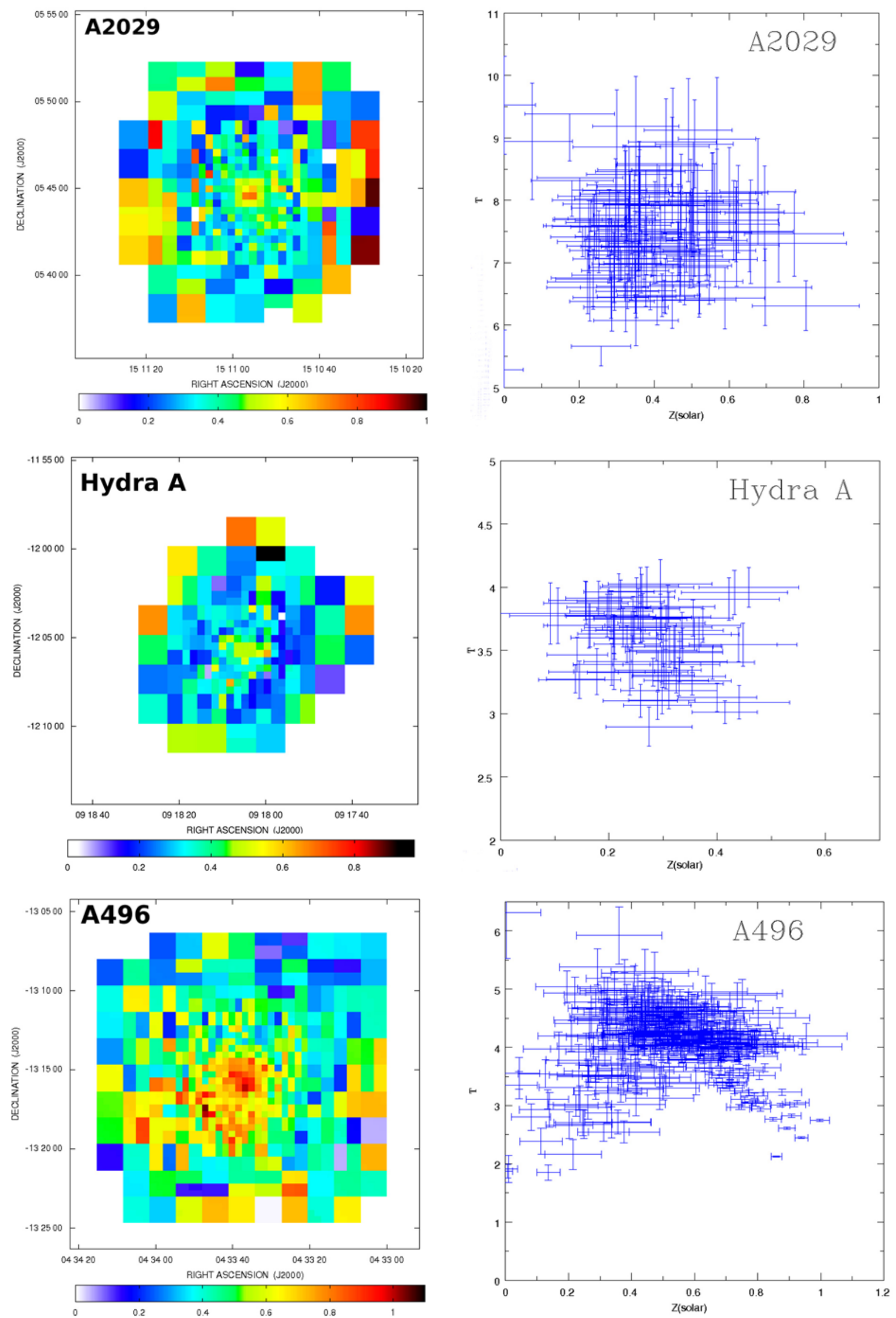

Fig. 1: left panels: Metallicity maps based on spectra from all three EPIC cameras, right panels: Plot of abundances against temperature for each bin 
gions for the map were selected following the method presented in [14], which we can summarize as follows: a square region centered on the X-ray peak was defined to include the area with high surface brightness. The region size of the pixels was optimized to be as small as possible by splitting it into horizontal or vertical segments through its center, while including at least 5000 source counts.

The metallicity distribution appears clearly nonspherical. In Fig. 1 (left panels) we show 3 examples of the obtained metallicity maps. For Centaurus and Sérsic 159-03, there is a peak in the center and then it decreases in the outskirts, while A496 (as A2029 and Hydra A) shows high metallicity clumps both in the center and in the outskirts. Several clumps are not significant but most of them deviate significantly (99 \%c.l) from the average profile. We note that, since Centaurus is a very low redshift $(z=0.0114)$, in these observations we are looking in the very central part of the cluster $(r<200 \mathrm{kpc})$ compared with the others clusters, and this could explain its different shape. On the other hand, Sérsic, for which we map the metal distribution for more than $350 \mathrm{kpc}$, we observe the same shape as Centaurus. Several maxima are visible in the metal distribution, which are not associated with the cluster center. From simulations $[15,16]$, we know that the maxima are typically at places where galaxies just have lost a lot of gas due to ram pressure. Since the gas lost by galaxies is not mixed immediately with the ICM at the place where we observe a metal blob, we should also observe a low temperature due to the fact that the gas in galaxies is cooler than the ICM. We therefore produced temperature maps of the clusters with the same spatial resolution obtained for the metal maps, and then we plotted the abundance of bins against their temperature, see Fig. 1 (right panels). Since we are searching for cool high-metallicity clumps due to the ejection of gas from galaxies and not the cool high-metallicity bins found in the cool cores, we did not plot the inner bins. Apart from Centaurus, we see a deviation from the expected temperature-metallicity relation. Most of the deviating points corresponds to the locus of low metallicity and low kT. The deviation could be due to the iron bias effect that cause an underestimation of the metallicity when we attempt to fit with a single temperature model a plasma that is, in fact, characterized by a combination of different temperatures [17, 18]. On the other hand, in the range of temperature between 3 and $4 \mathrm{keV}$, the inverse iron bias effect, that cause an overestimation of the metal abundance [19], can play an important role. The combination of these two effects could explain the large scatter in the distribution of Fig. 1. It is also possible that the ejected gas, with $T<1 \mathrm{keV}$ and metallicity in the range $0.5-1.5[20,21]$, will be heated up to the temperature of the surrounding gas (ICM) on a shorter time-scale than the time-scale of metal mixing. In this case, after a while we should observe a region of high metallicity (not yet dispersed) and high temperature (heated up at ICM temperature). Another possible explanation for the spread in the distribution could be related to the number of intracluster supernovae. During ram-pressure stripping events, many stars in fact form in the tail of stripped gas. The stars evolve and explode as $\mathrm{SNe}$ directly in the ICM and they can enrich the ICM very efficiently. In this case we should see clumps of high metallicity (due to SNe explosions) and high temperature. Obviously, more complex heating and cooling processes are at work, thus the simple picture of stripped gas does not hold.

\section{Conclusion}

Based on XMM-Newton observations, we have studied the spatial distribution of metal abundances in a sample of 5 relaxed clusters. We found that even for relaxed clusters the distribution of metals is clearly non-spherical. It appears very inhomogeneous, with several maxima separated by low metallicity regions. Moreover, we found a deviation from the temperature-metallicity anti-correlation that can be partially explained by the iron bias and inverse iron bias effect. However, a set of simulations that takes into account thermal conduction should be performed to better understand the physics of the IntraCluster Medium.

\section{References}

[1] Schindler, S., Diaferio, A.: Metal Enrichment Processes, Space Science Reviews, 2008.

[2] Kapferer, W., Knapp, A., Schindler, S., Kimeswenger, S., van Kampen, E.: Star formation rates and mass distributions in interacting galaxies. $A \& G$ A 438, 2005, p. 87.

[3] Kapferer, W., Ferrari, C., Domainko, W., Mair, M., Kronberger, T., Schindler, S., Kimeswenger, S., van Kampen, E., Breitschwerdt, D., Ruffert, M.: Simulations of galactic winds and starbursts in galaxy clusters, $A \& \mathcal{E} A, \mathbf{4 4 7}, 2006$, p. 827 .

[4] De Grandi, S., Ettori, S., Longhetti, M., Molendi, S.: On the iron content in rich nearby clusters of galaxies, $A \& \mathcal{E} A, \mathbf{4 1 9}, 2004$, p. 7 .

[5] Schmidt, R. W., Fabian, A. C., Sanders, J. S.: Chandra temperature and metallicity maps of the Perseus cluster core, MNRAS, 337, 2002, p. 71 . 
[6] Sanders, J. S., Fabian, A. C., Allen, S. W. Schmidt, R. W.: Mapping smallscale temperature and abundance structures in the core of the Perseus cluster, MNRAS, 349, 2004, p. 952.

[7] Durret, F., Lima Neto, G. B., Forman, G. B.: An XMM-Newton view of the cluster of galaxies Abell 85, $A \&$ \& A32, 2005, p. 809.

[8] O’Sullivan, E., Vrtilek, J. M., Kempner, J. C., David, L. P., Houck, J. C.: AWM 4: an isothermal cluster observed with XMM-Newton, $M N$ RAS, 35\%, 2005, p. 1134.

[9] Sauvageot, J. L., Belsole, E., Pratt, G. W.: The late merging phase of a galaxy cluster: XMM EPIC observations of A 3266, $A \& A, 444,2005$, p. 673 .

[10] Werner, N., de Plaa, J., Kaastra, J. S., Vink, J., Bleeker, J. A. M., Tamura, T., Peterson, J. R., Verbunt, F.: XMM-Newton spectroscopy of the cluster of galaxies $2 \mathrm{~A} 0335+096, A \& \mathcal{E} A \mathbf{4 4 9}$, 2006 , p. 475.

[11] Sanders, J. S., Fabian, A. C.: Enrichment in the Centaurus cluster of galaxies, $M N R A S, \mathbf{3 7 1}$, 2006, p. 1496 .

[12] Hayakawa, A., Hoshino, A., Ishida, M., Furusho, T., Yamasaki, N. Y., Ohashi, T.: Detailed XMM-Newton Observation of the Cluster of Galaxies Abell 1060, PASJ, 58, 2006, p. 695.

[13] Simionescu, A., Werner, N., Böhringer, H., Kaastra, J. S., Finoguenov, A., Brüggen, M., Nulsen, P. E. J.: Chemical enrichment in the cluster of galaxies Hydra A, $A \& \&$ A, 493, 2009, p. 409 .

[14] Lovisari, L., Kapferer, W., Schindler, S., Ferrari, C.: Metallicity map of the galaxy cluster A3667, $A \&$ \& $A$, 508, 2009, p. 191.
[15] Kapferer, W., Kronberger, T., Ferrari, C., Riser, T., Schindler, S.: On the influence of rampressure stripping on interacting galaxies in clusters, MNRAS, 389, 2008, p. 1405.

[16] Kapferer, W., Sluka, C., Schindler, S., Ferrari, C., Ziegler, B.: The effect of ram-pressure on the star formation, mass distribution and morphology of galaxies: $A \mathscr{E} A, \mathbf{4 9 9}, 2009$, p. 87 .

[17] Buote, D. A.: X-ray evidence for multiphase hot gas with nearly solar Fe abundances in the brightest groups of galaxies, $M N R A S, \mathbf{3 1 1}$, 2000, p. 176.

[18] Rasia, E., Mazzotta, P., Bourdin, H., Borgani, S., Tornatore, L., Ettori, S., Dolag, K., Moscardini, L.: X-MAS2: Study Systematics on the ICM Metallicity Measurements, ApJ, 674, 2008 , p. 728 .

[19] Gastaldello, F., Ettori, S., Balestra, I., Brighenti, F., Buote, D. A., De Grandi, S., Ghizzardi, S., Gitti, M., Tozzi, P.: Apparent high metallicity in 3-4 keV galaxy clusters: the inverse iron-bias in action in the case of the merging cluster Abell 2028, 2010, arXiv:1006.3255.

[20] Matsushita, K., Ohashi, T., Makishima, K.: Metal Abundances in the Hot Interstellar Medium in Early-Type Galaxies Observed with ASCA, PASJ, 52, 2000, p. 685.

[21] Athey, A. E., Bregman, J. N.: Oxygen Metallicity Determinations from Optical Emission Lines in Early-Type Galaxies, ApJ, 696, 2009, p. 681.

Lorenzo Lovisari

Sabine Schindler

Wolfgang Kapferer

E-mail: Lorenzo.Lovisari@uibk.ac.at

Institute of Astro-and Particle Physics

Technikerstrasse 25, A-6020 Innsbruck, Austria 